\title{
CORPI GEOPOLITICI: CONFINI ITALIANI A SUD E A EST, FRONTIERE SESSUATE E NARRAZIONI TRANSNAZIONALI
}

\author{
Les ÎLES POSTEXOTIQUES ${ }^{1}$
}

\begin{abstract}
A partire dalle storie del secondo dopoguerra di territori frontalieri a Nord-Est e a Sud d'Italia, ci proponiamo di riattraversare la geografia simbolica dello Stato italiano e di trasformare i concetti di Sud, Est, periferia, frontiera, per come sono stati prodotti e utilizzati dalle autorità accademiche e istituzionali. Assumere un posizionamento minoritario permette di riconoscere la propria posizione strategica e di contraddire la pressione virile del/la capitale. La messa in relazione delle storie di territori frontalieri evidenzia la necessità di decostruire le divisioni (Nord/Sud, Est/Ovest, uomo/donna, centro/periferia...) che marcano profondamente gli immaginari collettivi e dis-orientano, incitando a nuovi spostamenti verso le/i capitali/e del Nord e Ovest Europa.
\end{abstract}

PAROLE ChIAvE: Frontiere sessopolitiche, Ovest/Est Europa, Nord/Sud Italia, pratiche artistiche transnazionali, autoantropologia.

Cuerpos geopolíticos: límites del Sur y del Este de Italia, fronteras sexuadas y prácticas transnacionales

A partir de las historias posteriores a la segunda guerra mundial de los territorios fronterizos del Nordeste y del Sur de Italia, nos proponemos atravesar la geografía simbólica del Estado italiano y transformar las ideas del Sur, del Este, de la periferia y de la frontera que se han producido y utilizado por las autoridades institucionales y académicas. Asumir una posición minoritaria nos permite una posición estratégica en contradicción a la presión viril del/de la capital. La puesta en relación entre las historias de territorios fronterizos acentúa la necesidad de deconstrucción de las divisiones (Norte/Sur, Oeste/Este, hombre/mujer, centro/periferia...) que marcan profundamente los imaginarios colectivos y des-orientan, provocando nuevos desplazamientos cerca de los/las capitales del Norte y del Oeste de Europa.

\footnotetext{
1 "les îles postexotiques" è un progetto transnazionale-sessuale-linguistico-frontaliero, nato da una collaborazione antropologico-artistica e da una corrispondenza di immaginari e di sogni tra delle isole. Si tratta di arcipelaghi in lotta, di luoghi creativi, umidi e collettivi che sono stati immaginati nel 2013 tra Verona (Italia) e Valencia (Spagna). (lesilespostexotiques@gmail.com)
} 
PALABRAS Clave: Fronteras sexopolíticas, Oeste/Este Europa, Norte/Sur Italia, practicas artísticas transnacionales, autoantropología.

Geo-political bodies: Italy's South-Eastern borders, sexualized frontiers and transnational narratives

Starting from post-WWII stories of North-Eastern and Southern borderlands, we set out to review the symbolic geography of the Italian state and transform the concepts of 'South', 'East', 'periphery' and 'frontier', as produced and used by institutional and academic authorities. Taking up a minority positionality allows recognising one's own strategic place, and contradicting the virile pressure of (the) Capital. Linking together the stories of borderlands highlights the need for deconstructing those divisions (North/South, West/East, man/woman, core/periphery, etc.) that deeply mark collective imaginaries and dis-orient subjects, urging them to relocate in the Capital(s) of North-Western Europe.

KEY WORDS: Sexpolitics borders, Western/Eastern Europe, Nothern/Southern Italy, trasnational artistic practices, autoanthropology.

In Europa le frontiere italiane a est e a sud fungono da cerniera e incarnano la spartizione: ad est dividono l'est post-socialista dall'ovest democratico-capitalista e a sud creano una strettoia tra occidente europeo, Medio Oriente e Africa.

Per analizzare i processi socio-economici e culturali conflittuali che attraversano lo Stato e l'identità italiana partiremo dunque dalle analogie e dalle corrispondenze rintracciabili tra l'est e il sud. Interpellando questi confini, ci proponiamo di riattraversare la geografia simbolica della Repubblica e di questionare i concetti di sud, est, periferia e frontiera che la costituiscono.

In primo luogo connetteremo l'ideologia della nazione con le dinamiche di etnicizzazione e omologazione etnica determinate dal consolidamento a partire dal secondo dopoguerra dell'Italia in quanto Stato nazionale; benché sia stata storicamente una potenza colonizzatrice e un paese allineato con il blocco occidentale, le storie delle sue zone di frontiera a sud e a nordest hanno riflesso le transizioni postcoloniali e postsocialiste.

Proponiamo qui di pensare lo Stato come un corpo e di assumere un posizionamento frontaliero, al fine di contraddire la pressione virile del/la capitale sui margini della nazione. Approfondiremo la relazione ovest/est che intercorse tra Italia e Jugoslavia attraverso una prospettiva sesso-genere, comparandola alla relazione tra $\mathrm{i}$ due poli nord e sud Italia. Discutere l'opposizione uomo/donna in relazione alla geopolitica consente sia di autocriticare le dinamiche di potere che legano queste due figure, quanto due Stati, ma anche di mettere in luce le loro differenti costituzioni, entrambe escludenti e orientate, pur se in modi diversi, al controllo di corpi e territori.

Con l'intenzione di contrastare l'egemonia geopolitica-sessuale del/la capitale analizzeremo la costruzione del confine tra Italia e Jugoslavia attraverso le storie dei territori dell'Adriatico nordorientale, un'area geografica di carattere plurinazionale e translinguista che oggi si trova tra Italia, Slovenia e Croazia. 
A sud il punto di vista sarà la Sicilia, un esempio di isola-frontiera dove hanno avuto e continuano ad avere luogo dei processi che ricalcano dinamiche socio-economiche e rappresentazioni culturali neo e postcoloniali; guardare l'Italia dall'estremo sud è un incoraggiamento a liberare i sud dalla presenza fondante di uno o tanti nord e a vivere all'interno di un paradigma dell'autonomia che favorisca piuttosto le relazioni oblique come quella qui proposta, che considera le storie del sud e dell'est in connessione.

Scegliamo questi territori perché li conosciamo per biografia e per genealogia, convinte che pratiche autoetnografiche (Reed-Danahay, 1997) condivise possano rappresentare dei punti di vista capaci d'incoraggiare nuovi passaggi. Mettere in corrispondenza narrazioni considerate di norma disgiunte e attivare processi di traduzione di contesti sono le pratiche politiche da cui prende vita questo articolo; il fine è mettere in evidenza la necessità di decostruire i binarismi e le relazioni di potere che marcano profondamente gli immaginari collettivi geopolitici ed incitano a spostamenti verso le/i capitali del nord-ovest Italia ed Europa come verso identità sessuali definite da un sistema di privilegi patriarcali, etnici e di classe, su cui si fondano le democrazie europee contemporanee.

\section{Vestiti di bandiere}

Fu la domenica mattina verso le sette che gli americani entrarono coi camion, tra due file di stracci bianchi che sventolavamo dai lati della strada [...] quando la prima macchina fu sotto il balcone, buttai i fiori gridando: viva gli americani [...] alcuni soldati tedeschi piangevano e si nascondevano tra la folla. Ad uno diedi un vecchio paio di pantaloni e un giubbotto di mio marito, altre donne seguirono il mio esempio e i tedeschi travestiti si poterono allontanare confondendosi coi borghesi, mentre gli americani occupavano la città. La speranza si accese nel cuore del popolo. Si pensava che finalmente ci sarebbe stata giustizia anche per i poveri, che la guerra finiva presto e che la pace era dietro alla porta. (Occhipinti, 1976: 67-68)

Invece nell'immediato secondo dopoguerra in Sicilia, l'ordine geopolitico bipolare instauratosi a causa della sfida tra le potenze statunitensi e sovietiche, e l'esigenza di scongiurare il pericolo rosso, accelerarono il ristabilimento degli equilibri di forza esistenti durante il periodo fascista. Come ci racconta $\mathrm{M}$. Occhipinti, il governatore americano appena arrivato fece degli accordi con i fascisti che continuarono a gestire le amministrazioni a proprio favore e chi era appena tornato dalla guerra ricevette le cartoline rosa per l'arruolamento a sostegno della lotta antifascista al Nord. Il popolo non rispose però all'appello all'identità italiana. L'azione di disobbedienza civile a Ragusa contro la coscrizione obbligatoria, spesso ridotta ad un rigurgito di fascismo o a sussulti 
del movimento separatista, dal punto di vista di Maria, comunista non allineata con il fervore patriottico del partito e organizzatrice della resistenza, si era trattato piuttosto di una "ribellione dei giovani spontanea e sincera", perché non si poteva parlare di guerra a "gente che dopo un anno e mezzo di occupazione alleata non credeva più nella patria” (Occhipinti, 1976: 94). L'onore e l'interesse d'Italia si tradussero per una parte della popolazione in rastrellamento dei renitenti, stragi e prigionia, suggellando uno dei paradossi dell'appartenenza nazionale ad uno Stato antifascista; questo fu anche l'esito dell'episodio considerato fondante il movimento femminista in Sicilia e che ebbe nel movimento di occupazione delle terre il suo seguito e le sue attiviste (Mafai, 1976).

Che non si tornasse sempre indietro d'altra parte dalla guerra è noto; nella famiglia di mia nonna ad esempio, fu il fratello più piccolo a restare disperso, nonostante una sua lettera a matita annunciasse il rientro ben presto. Diceva di essersi rifugiato in Jugoslavia in casa di una ragazza straniera con cui aveva progettato un viaggio al Sud. Il padre aveva mandato a comprare il letto ed era pronta la stanza del balcone, ma dopo mesi di attesa, Nuccio, un commilitone figlio dei gelatai del paese, disse che in un campo di vigne, tornando, era saltato in pezzi su una mina.

"Era settembre e io ero andata a Cherso da questa famiglia di amici per vendemmiare l'uva. L'8 ci fu l'armistizio e ricordo che la gente dava vestiti ai soldati italiani. Da noi c'era un soldato di Ancona, è stato lì per due tre giorni e per scappare si è messo i vestiti civili che gli hanno dato. E questo nel '43, quando io avevo 11 anni". ${ }^{2}$ Anche qui dovevano entrare gli americani, invece al loro posto sono arrivati i tedeschi.

Mia nonna racconta "eravamo a Privlaka e abbiamo fermato un camion di tedeschi per chiedergli se ci portavano fino a Lussino e ci hanno preso, pensa che matti: loro andavano a occupare e noi in questo camion. Non sapevano parlare italiano e quando siamo arrivati in Riva era tutto pien de cucine da campo! Poi una volta a casa la mamma mi ha detto che le avevano dato piselli, pasta, riso e tutti mangiavano perché gliel'avevano portato questi tedeschi!"3

In seguito disseminarono di mine il molo dicendo che prima di scappare avrebbero fatto esplodere tutto e invece, ricorda, un uomo di Lussino tagliò dei cavi così il paese si salvò e i partigiani lo festeggiarono come un eroe.

La sconfitta dell'Italia fascista nella seconda guerra pone fine alla mira di farne una potenza mondiale, determinerà l'epilogo del potere fascista su tutte le colonie e, rispetto al confine nordorientale, la cessione alla Jugoslavia di FiumeRijeka, del territorio di Zara-Zadar, delle isole di Lagosta-Lastovo e Pelagosa-

\footnotetext{
${ }^{2}$ Raccolta, S. Intervista telefonica da Jordana. 6/11/2011.

${ }^{3}$ Ibidem.
} 
Pelagruža, di gran parte dell'Istria-Istra, del Carso-Kras triestino e goriziano e dell'alta valle dell'Isonzo-Soča.

Si riunisce nel '46 la Commissione confini e vengono proposte sette nuove linee di demarcazione tra Italia e Jugoslavia. La linea Morgan, ufficiale britannico delle truppe alleate in Italia, decise il confine provvisorio tra i due Stati, non tenendo conto delle realtà nazionali né degli interessi delle popolazioni locali.

Nella prospettiva occidentale si trattava di negoziare con l'Unione Sovietica un equilibrio planetario basato sul concetto di opposizione bipolare. L'Italia diventa uno Stato cuscinetto tra occidente e blocco socialista: esistono due mondi che si fronteggiano a livello locale e influenzano a livello globale orientamenti politici promuovendo l'applicazione del binarismo ad ogni settore della vita.

La stessa logica s'impone intanto a sud, si trasforma adattandosi al contesto e, producendo nuove dicotomie, influenza in modo decisivo gli avvenimenti storici del dopoguerra in Sicilia. In questo senso, R. Scarpinato interpreta l'eccidio di Portella della Ginestra nel '47 come una strage il cui obiettivo era smorzare la vittoria del fronte socialista-comunista, "il punto zero della strategia della tensione che nei decenni successivi insanguinerà il Paese assemblando in un unico blocco criminale le parti più retrive della classe dirigente e apparati di potenze straniere" (Lodato \& Scarpinato, 2008: 221).

Contemporaneamente a livello ideologico e culturale si reinnesta nella storia la questione meridionale, nata alle soglie dell'unificazione d'Italia, sotto forma dapprima di una tensione verso la modernizzazione del paese, in un secondo momento invece dell'inveramento di un Sud arretrato. Nel ' 47 fu sancito lo Statuto siciliano di cui uno dei principi saldi era la tesi "riparazionista" nittiana, che si concretizzò nell'istituzione di un fondo di solidarietà nazionale grazie al quale si sarebbero dovuti pareggiare i minori redditi della Sicilia. Nonostante le intenzioni dei proponenti, la qualificazione "riparazionista" riporta al campo semantico della violenza dello stupro, svelando quale potesse essere il substrato ideale della relazione tra il sud e il/la capitale. Mentre le donne iniziano a liberarsi attraverso atti coraggiosi come quello di una ragazza di Alcamo, Francesca Viola, la prima a rifiutare il matrimonio "riparatore" nel '65, il linguaggio istituzionale persiste nel lessico e nella logica patriarcale: il governo siciliano si presta al processo di femminilizzazione e al connubio politico con lo Stato centrale.

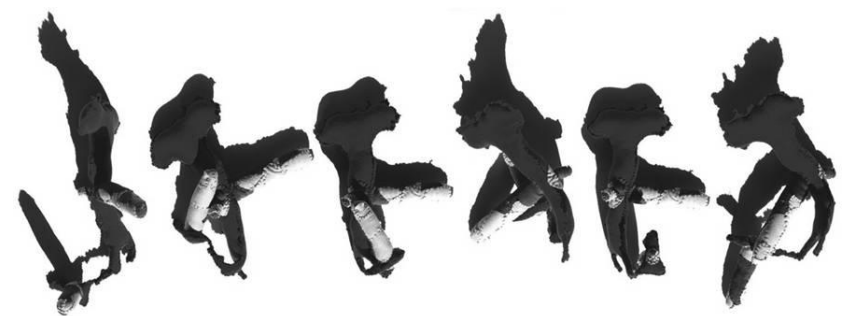

Mappe nazionali cucite, Roma e Palermo, città segnate da capezzoli e dita. Gioco sulla relazione politico-sessuale tra penisola italiana e Sicilia, 1861-2012. 
La maggior parte della produzione antropologica dell'epoca, italiana e anglofona, contribuisce al processo di etnicizzazione del sud Italia, rappresentandolo attraverso la lente tradizione-modernità. L'immagine che viene più diffusa si basa sulla nozione di "familismo amorale" inteso come un sistema che, privilegiando l'interesse della famiglia nucleare e il controllo sulla riproduzione, esclude i valori della cooperazione e della comunità e fonda l'arretratezza.

Anche a livello economico negli anni ' 50 si registra un cambiamento; secondo la storica L. D'Antone infatti fino ad allora "il sud italiano non si poteva definire corpo economico-sociale con dinamiche economiche, istituzionali e sociali radicalmente diverse da quelle del resto del paese" (2009: 278), ma fu durante quegli anni che l'azione governativa centrale e la retorica del meridionalismo hanno prodotto il "Mezzogiorno arretrato" con contributi a fondo perduto, sgravi fiscali, zone di sviluppo industriale che resero la regione un'area di attrazione per imprenditori e politici spregiudicati del nord, del centro e del sud che si integrarono nei vari apparati governativi.

Mentre s'installa il pensiero neocoloniale e la Sicilia si puntella di avamposti militari e basi nato, mentre prende avvio il primo polo di raffinazione petrolifera nella zona sudorientale eppure l'emigrazione continua a costituire una delle principali risorse economiche, si avvertono anche in Sicilia gli effetti della diaspora causata da una comparabile dinamica etnicizzante vissuta sul confine a nordest.

Furono allestiti in Sicilia 5 dei 120 Campi Raccolta Profughi che accolsero legli esuli istriane-i, giuliane-i e dalmate-i; per non consentire alle comunità di riunirsi facilmente i campi erano disseminati su tutto il territorio nazionale ed erano ricavati da caserme e stabilimenti industriali dismessi, manicomi, scuole, conventi, ospedali in cui trovarono alloggio anche profughe-i italiane-i provenienti da Grecia e Tunisia (Pupo, 2005: 209).

Mia nonna arriva nel giugno del '47 ai Silos di Trieste, rimane a lavorare in città come balia, ha 15 anni, e nel settembre dello stesso anno raggiunge la sua famiglia al campo profughi di Fesca, in provincia di Bari, dove visse per 8 anni. Era un'ex colonia marina fascista, un edificio di due piani che dava sul mare; nelle finestre della stanza si fermavano col vento le alghe e a loro sembrava di vivere su una nave.

Nel campo convivevano circa 200 famiglie, imparò a cucire da una signora greca e ad andare in bicicletta mentre Franco il postino consegnava la posta o con la vecchia bici militare del lattaio, con il pignone fisso e le ruote piene. Non c'era altro da fare e non c'erano soldi, in aprile erano già tutte-i abbronzate-i, facevano il bagno e poi salivano nella grande terrazza ad asciugarsi.

Intanto Trieste, l'estremità, diviene simbolo del completamento dell'unità nazionale. Tra il 1944 e la fine degli anni ' 50 più di 250 mila persone, in massima parte italiane, abbandonarono le città di Zara, Fiume, le isole di Cherso e Lussino 
e la penisola istriana. Esodarono tutte e tutti quelli che diffidarono della nuova amministrazione jugoslava, molte-i si stabilirono in Italia mentre altri proseguirono verso Australia, Stati Uniti, Canada, Venezuela, Argentina, Brasile e Sudafrica (2005: 234).

Il punto di vista qui espresso dipende dal mio posizionamento in quanto terza generazione, dai racconti familiari sull'esodo e dall'aver vissuto il confine italo-jugoslavo, frontiera ideologica e linguistica che ho attraversato dal '78 e che $\mathrm{mi}$ ha spinta a cercare un'interpretazione alternativa a quelle delle due parti opposte. I racconti familiari s'incrociano con la Storia ufficiale, fondamento della nazione: la famiglia di mia madre ha vissuto l'esodo, stabilendosi infine a Roma, mentre quella di mio padre non si è spostata mai dal proprio contesto d'origine.

Ritrovare delle corrispondenze tra lo scarto maschile/femminile di queste due diverse storie e i governi subentrati nei territori in questione, hanno segnato la prospettiva di cui oggi mi avvalgo per leggere le coincidenze tra geopolitica, sessualità e genere. E' certo che chi guarda dall'esterno avrà sempre uno sguardo parziale o, come è successo a me guardando l'ex Jugoslavia dal confine orientale italiano, sarà sempre uno sguardo interrotto.

La gerarchia e la minaccia della violenza sono caratteristiche della costituzione di ogni potere centrale ed interessano sia la democrazia italiana che il socialismo autogestito jugoslavo. Nonostante la struttura patriarcale sia stata parte fondante della memoria nazionale e del partito ${ }^{4}$, la ex Jugoslavia rappresentava a livello internazionale, nei rapporti con l'Italia, l'incarnazione del sistema opposto. La caratteristica comune richiesta ad uno Stato, affinché possa instaurare diversi rapporti di potere economici e politici con altri Stati più o meno potenti, è la versatilità; le identità politiche di Italia e Jugoslavia di cui ci serviamo nell'analisi non possono essere considerate dunque caratteristiche fisse.

L'esodo giuliano dalmata definisce un movimento, un desiderio di singoli e masse di sfuggire al nemico cioè all'opposto. Si è trattato di fuggire alla declassazione di un gruppo nazionale precedentemente in lotta per essere riconosciuto come dominante e, rispetto ai rapporti internazionali tra gli Stati, di sfuggire alla posizione subalterna (in relazione all'occidente) che avrebbe incarnato un'intera società civile, una volta passata sotto il controllo del blocco sovietico.

L'analisi degli eventi diasporici che hanno marcato l'Adriatico nordorientale (Cattaruzza, 2003) ci è utile nella (auto)critica alla costruzione di identità fisse nazionali, linguistiche e sessuali basate sulla divisione del mondo in due macro aree di influenza.

In modo particolare la via dell'esilio percorre la separazione da un corpo, territorio, governo, sesso, verso una trasformazione. La comparazione tra exil e

\footnotetext{
${ }^{4}$ Generazione jugoslava I e II, intervista di A. Rossini a R. Iveković, Osservatorio Balcani e Caucaso 10-14/08/2007.
} 
transizione è possibile dunque data la fuga comune da un sistema identitario chiuso. Proponiamo perciò di pensare in termini di gubitak/pirdiri/perdita come condizione strategica minoritaria e frontaliera, punto di partenza valido per pensare e vivere territorio e corpo attraverso esperienze e prospettive dislocate.

Se consideriamo l'identità di genere ${ }^{5}$ in quanto forma di governo, si possono immaginare delle trasformazioni di tipo transgenere in spazi geografici in cui si sono avvicendati diversi poteri. Le minoranze etniche, in questi processi, incarnano la presenza in un corpo sessuato di parti associate di norma al sessoPaese opposto o subalterno.

L'esodo giuliano dalmata pertanto si può considerare un desiderio di mascolinizzazione o di opposizione alla femminilizzazione forzata cui viene subordinato un intero territorio che, dopo la seconda guerra mondiale, passa da fascismo a socialismo "cambiando" perciò identità di genere.

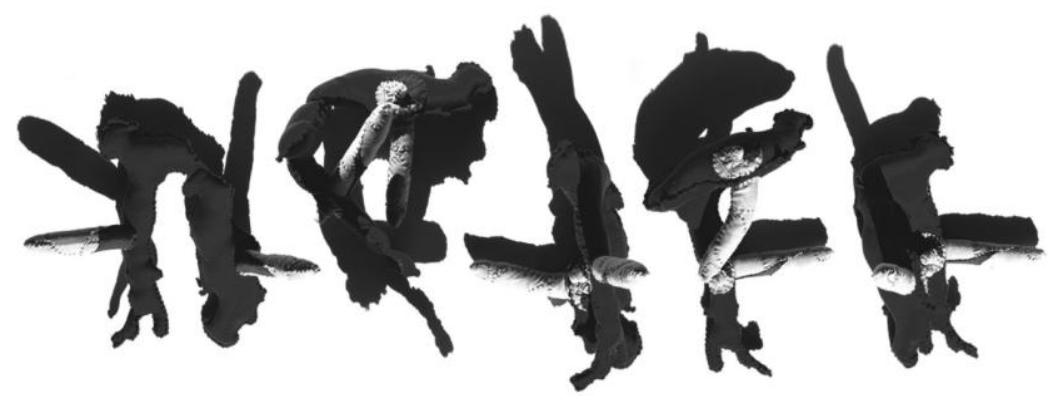

Mappe nazionali cucite, capitali segnate da capezzoli e dita. Gioco sulla relazione politico-sessuale tra Italia ed ex Jugoslavia, Roma-Belgrado, 19451992

Dopo il 1989 il processo di europeizzazione subisce un'accelerazione che determina nuove frontiere, zone riservate alla violenza e forme ulteriori di etnicizzazione: ha inizio la guerra in ex Jugoslavia, in Sicilia ha culmine la stagione stragista negli attentati ai giudici Falcone e Borsellino e l'immigrazione dai Paesi dell'est, dal Nordafrica e dall'Albania, viene rappresentata sempre più come un problema di sicurezza.

Dal 1980 al '92 il sud Italia è marcato dalla strategia del terrore finalizzata a creare una frattura Nord-Sud con l'uso destabilizzante della violenza. Per chi come me è nata a Palermo nei primi anni ' 80 ed ha partecipato da bambina alla nascita del movimento antimafia, vivere al Sud significa ritrovarsi con questa eredità storica da interpretare, con i sensi di colpa, i complessi d'inferiorità, il

\footnotetext{
${ }^{5}$ Costruzione socio-culturale attraverso cui gli individui s'identificano o vengono identificati secondo le categorie binarie e identitarie di maschile o femminile.
} 
desiderio di riscatto, di fuga e il senso di forte limitazione del possibile che ne derivano. L'interpretazione dei meccanismi della violenza affidata ad ogni cittadino e alla magistratura, che ha svelato il carattere fictionnel della retorica dei "martiri di Stato" e dello "Stato-difensore" tanto quanto della contrapposizione tra mafia e Stato, ha offerto una possibilità di decifrare l'inganno dell'educazione alla legalità, promossa dalle stesse istituzioni che producono e diffondono violenza e gerarchia.

Un posizionamento che assume questi punti di partenza per guardare al sud non vi ritrova più una condizione patologica endemica di arretratezza ma intuisce le reti invisibili di potere che lo attraversano e ne determinano le condizioni socio-economiche, un immaginario contaminato rispetto a ecologia ed economie - anche non monetarie- possibili. Collocare il Sud in questo quadro ne chiarisce la posizione all'interno delle dinamiche di potere nazionali e transnazionali che lo attraversano, ma non offre un orizzonte di pensiero sufficientemente efficace per chi lo abita e cerca di innestare conflitti e pratiche di vivibilità (partecipazione associativa, ecosostenibilità, autorganizzazione cittadina ecc.). Non offre chiavi di lettura e di azione neppure un rinnovato immaginario apparentemente postcoloniale che rivela l'altra faccia della stessa medaglia, ovvero la visione di un sud idealizzato e salvifico, un paradiso inconsistente nel quotidiano.

Nei tentativi svariati di abitare il/al sud, in questo passaggio biografico, ritrovo la possibilità di prenderlo a risorsa per una diversa visione del presente $\mathrm{e}$ delle possibilità di trasformazione dei contesti: contaminare le narrazioni sul sud con altre narrazioni, per rendere evidente che non vive in una condizione isolata né incancrenita, il cui riscatto non proviene da un'esaltazione della marginalità ma dalla connessione con altre storie, perché come scrive F. Cassano "ogni sud che per salvarsi si sgancia dagli altri sud cede alla tentazione di farsi cooptare" (Cassano, 2009: 57). Così tentiamo di vivere il Sud e l'Est come non "ci" sono stati raccontati e di tracciare in modo netto le coordinate anarchiche ${ }^{6}$ che li renderebbero luoghi vivibili.

\section{TESTI CITATI}

Cassano, Franco (2009), “Tre modi di vedere il Sud”, I Sud. Conoscere, cambiare, capire, Marta Petrusewicz, Jane Schneider e Peter Schneider (eds.), Bologna, il Mulino.

\footnotetext{
${ }^{6}$ Cfr. Intervento di Antonietta Potente. ¿...cuándo? Ahora... le anarchiche coordinate del tempo politico-esistenziale. Seminario di Diotima 2011: La festa è qui. Università di Verona. 11 novembre 2011.
} 
Cattaruzza, Marina (ed.) (2003), Nazionalismi di frontiera. Identità contrapposte sull'Adriatico nord-orientale 1850-1950, Soveria Mannelli, Rubbettino.

D’Antone, Leandra (2009), "Studiare l'Italia nel Sud: una riflessione personale”, I Sud. Conoscere, cambiare, capire, Marta Petrusewicz, Jane Schneider e Peter Schneider (eds.), Bologna, il Mulino.

Lodato, Saverio e Roberto Scarpinato (2008), Il ritorno del principe, Milano, Chiarelettere.

Mafai, Simona (ed.) (1976), Essere donna in Sicilia, Roma, Editori Riuniti.

Occhipinti, Maria (1976), Una donna di Ragusa, Milano, Feltrinelli.

Pupo, Raoul (2005), Il lungo esodo, Milano, Rizzoli.

Reed-Danahay, Deborah (ed.) (1997), Auto/Ethnography: Rewriting the Self and the Social, Oxford, Berg. 